\title{
Variabilité du taux des lymphocytes CD4 et de la charge virale chez les personnes vivant avec le VIH sous traitement antiretroviral : cas de l'hopital saint Jean De Dieu de Tanguieta (Benin)
}

\author{
Evelyne LOZÈS ${ }^{1 *}$, Clément AHOUSSINOU ${ }^{2}$, \\ Micheline AGASSOUNON TCHIBOZO DJIKPO ${ }^{3}$, Edwige DAHOUEGNON ${ }^{4}$, \\ Nanette AHOSSOUHE ${ }^{1}$, Arnaud ACOTY ${ }^{2}$ et Comlan de SOUZA ${ }^{5}$ \\ ${ }^{1}$ Université d'Abomey-Calavi, Ecole Polytechnique d'Abomey-Calavi, Laboratoire de Recherche en Biologie \\ Appliquée UR-SBME, 08 BP 691CTP Cotonou/Bénin. \\ ${ }^{2}$ Programme National de Lutte Contre le VIH-SIDA, Ministère de la Santé, Bénin. \\ ${ }^{3}$ Université d'Abomey-Calavi, Faculté des Sciences et Techniques, 01 BP1636RP Cotonou/Bénin. \\ ${ }^{4}$ Département : Génie des Technologies Alimentaires, Ecole Polytechnique d'Abomey-Calavi, \\ Université d'Abomey-Calavi, Bénin. \\ ${ }^{5}$ Ecole Supérieure des Techniques Biologiques et Alimentaires (ESTBA-UL), Université de Lomé, \\ BP 1515, Lomé, Togo. \\ *Auteur correspondant ; E-mail: lozes56@yahoo.fr; josette.lozes@epac.uac.bj
}

\section{RESUME}

L'objectif de cette étude était de mesurer l'impact des traitements antirétroviraux d'importation sur la charge virale et sur le taux des lymphocytes CD4 des personnes vivant avec le Virus de l'Immunodéficience Humaine (PV VIH) en phase du syndrome de l'immunodéficience acquise (SIDA) de l'hôpital de Tanguiéta au Bénin. L'étude était de type transversal, l'échantillonnage exhaustif avec recrutement de tous les malades sous traitement antirétroviral répondant aux critères d'inclusion de l'étude. La durée de l'étude était de 9 mois et la taille de l'échantillon de 94 patients. Les données collectées ont été contrôlées et saisies à l'aide du logiciel SPSS 15.0 For Windows et les tableaux réalisés après conception d'un programme d'analyse. Les résultats obtenus ont montré que la charge virale après quatre mois de traitement devenait indétectable chez $50 \%$ des patients et que 79,70\% d'entre eux devenaient immunocompétents $(r=-0,5)$. Après douze mois de traitement, $83,30 \%$ et $86 \%$ des patients avaient une charge virale indétectable et recouvraient leur immunocompétence respectivement $(r=0,11)$. De l'analyse des données, il ressort que les antirétroviraux ont une efficacité sur la population étudiée. Le taux des lymphocytes CD4 et la charge virale évoluent en sens inverses au cours du traitement. L'utilisation du coefficient de corrélation $r$ permet de suivre l'efficacité de la thérapie antirétrovirale.

(C) 2012 International Formulae Group. All rights reserved.

Mots clés : ARV, CD4, PV VIH, coefficient de corrélation.

\section{INTRODUCTION}

Les traitements antirétroviraux, dirigés contre le virus de l'immunodéficience humaine, sont depuis quelques années administrés dans les pays en développement malgré un nombre encore limité de patients y

(C) 2012 International Formulae Group. All rights reserved. DOI : http://dx.doi.org/10.4314/ijbcs.v6i2.9 
ayant accès (Ford, 2012; Stringer et al., 2012). L'efficacité des polythérapies antirétrovirales a déjà été démontrée (Oette et al., 2012). Cette efficacité peut ne pas concerner tous les types de population si l'on tient compte de l'environnement différent des populations, de la variabilité génétique du virus ainsi que de celle de l'hérédité de l'homme lui même (Ghosn et Viard, 2012). Les lymphocytes CD4 sont des marqueurs essentiels du statut immunitaire. Ils facilitent et entretiennent la réponse immunitaire. Ils sont les principales cellules cibles du VIH et expriment à leur surface la molécule CD4 qui est un récepteur de haute affinité pour la glycoprotéine 120 (gp 120) de l'enveloppe membranaire du VIH. Leur taux a donc tendance à diminuer lors de l'évolution de l'infection et leur mesure évalue l'étendue du dommage immunologique déjà subi d'où son rôle de critère d'indication thérapeutique principal (Danel et al., 2012). La charge virale (CV) quant à elle, est la quantification de l'acide ribonucléique plasmatique du VIH. C'est actuellement la meilleure façon d'apprécier la réplication du VIH dans l'organisme. Cette charge augmente donc lorsque l'infection évolue sans traitement. Idéalement, un traitement antirétroviral devrait faire augmenter le taux des lymphocytes CD4 en même temps qu'il devrait abaisser la charge virale. Cependant, le succès immunologique, remontée du taux de lymphocytes CD4, n'est pas toujours accompagné d'un succès virologique, abaissement de la charge virale (Sahali et al., 2011). Au Bénin, aucune étude n'a été réalisée montrant l'effet des ARV à la fois sur le taux des lymphocytes CD4 et sur la multiplication du VIH. L'objectif de cette étude était d'évaluer l'impact des traitements antirétroviraux à la fois sur le système immunitaire et la multiplication du virus chez des PV VIH du Bénin, en l'occurrence les PV
VIH de la région nord Bénin, suivis à l'hôpital St Jean de Dieu de Tanguiéta.

\section{MATERIEL ET METHODES \\ Population d'étude}

La population d'étude était constituée de malades infectés par le VIH, en phase SIDA et sous antirétroviraux d'importation, suivis à l'hôpital de Tanguiéta du Bénin.

\section{Méthodologies}

Il s'agissait d'une étude de type transversal. Les patients des deux sexes ont été reçus lors des consultations médicales, des bilans de suivi et de la dispensation des ARV. Les malades coinfectés par Mycobacterium tuberculosis et le VIH de même que ceux qui n'étaient pas sous antirétroviraux ont été exclus de l'étude. L'échantillonnage était exhaustif et a porté sur 94 cas. Le but de l'étude a été expliqué à chaque patient au cours d'un entretien individuel réalisé, pour obtenir son consentement éclairé. Les patients étaient par la suite soumis à un prélèvement sanguin lors des bilans. Les renseignements cliniques et biologiques ont été documentés à partir des dossiers médicaux et des registres de laboratoire. L'observance thérapeutique de la prise des ARV a été évaluée par le médecin traitant. Les données ont été recueillies à partir d'un questionnaire. Deux types de variables ont été étudiés. La variable dépendante qu'est la charge virale et les variables indépendantes liées aux caractéristiques (âge/sexe, taux de CD4, observance thérapeutique). Les données collectées ont été analysées à l'aide du logiciel SPSS 15.0 for Windows. En se basant sur la classification rapportée par Danel et al. (2012) et sur les Politiques, Normes et Procédures Nationales pour la Prise en Charge des IST au Bénin (MSP/PNLS, 2004) les malades ont été classés en immunodéprimés sévères, modérés et immunocompétents d'après le taux de CD4. De même, ces malades ont été classés pour la 
charge virale, en charge virale indétectable, modérée et élevée. Les associations entre la variable dépendante et les variables indépendantes d'une part et les variables indépendantes entre elles d'autre part ont été recherchées à l'aide du test statistique de $\mathrm{Khi}^{2}$ avec un intervalle de confiance à $95 \%$. La relation entre le taux des CD4 et la charge virale a été étudiée grâce aux tests de corrélation. Ces tests de corrélation ont été appliqués après une sélection des patients qui avaient à la fois les résultats de la numération des CD4 et ceux de la mesure de la charge virale après quatre et douze mois de traitement antirétroviral. Cette étude s'inscrivait dans le suivi des PV VIH; les données biologiques ont donc été respectivement étudiées après 4 et 12 mois de traitement antirétroviral conformément aux Politiques, Normes et Procédures Nationales pour la Prise en Charge des IST au Bénin (MSP/PNLS, 2004).

\section{RESULTATS}

\section{Caractéristiques démographiques}

Sexe et âge

La répartition de l'échantillon était de $37,8 \%$ d'hommes pour $62,2 \%$ de femmes. Une PV VIH sur vingt avait moins de 15 ans. Le plus jeune patient avait 6 ans et le plus âgé 60 ans. Le Tableau 1 montre la répartition de l'échantillon par classes d'âge.

\section{Caractéristiques cliniques \\ Observance thérapeutique}

L'observance thérapeutique était de $64,6 \%$.

\section{Caractéristiques biologiques}

Elles regroupaient le type de $\mathrm{VIH}$; le taux de CD4 à l'initiation aux ARV; le taux de CD4 et la charge virale après 4 et 12 mois de traitement. $95,1 \%$ des PV VIH ont été infectées par le VIH-1 et le reste par le dual VIH-1 et VIH-2. Les Tableaux 2, 3 et 4 montrent le taux de CD4 des patients à l'initiation du traitement, à quatre et 12 mois, respectivement. Les Tableaux 5 et 6 montrent la charge virale à quatre et 12 mois, respectivement. La première colonne des Tableaux 2, 3 et 4 répartit, d'après le taux de CD4, du haut vers le bas, les PV VIH de l'étude en immunodéprimés sévères, immunodéprimés modérés et immunocompétents, respectivement. La première colonne des Tableaux 5 et 6 répartit, pour la charge virale, du haut vers le bas les charges virales indétectables, modérées et élevées.

Le taux des immunodéprimés passe de $87,7 \%$ à l'initiation à 20,3\% après quatre mois de traitement et à $14,0 \%$ après douze mois de traitement. Concomitamment (Tableau 6), plus des 4/5 PV VIH, possédant à la fois la mesure du taux de CD4 et celle de la charge virale, présentent une charge virale indétectable après douze mois de traitement.

Tableau 1 : Répartition des PV VIH en fonction de l'âge.

\begin{tabular}{|c|c|c|}
\hline $\begin{array}{l}\text { AGE } \\
\text { (année) }\end{array}$ & $\begin{array}{l}\text { Effectif } \\
(N=82)\end{array}$ & $\begin{array}{c}\text { Fréquence } \\
(\%)\end{array}$ \\
\hline$<15$ ans & 4 & 4,9 \\
\hline $15-25$ & 1 & 1,2 \\
\hline $25-35$ & 28 & 34,1 \\
\hline $35-45$ & 30 & 36,6 \\
\hline$\geq 45$ & 19 & 23,2 \\
\hline
\end{tabular}


Tableau 2 : Répartition des PV VIH en fonction du taux de CD4 initial.

\begin{tabular}{lcc}
\hline CD4 en cellules/ $\boldsymbol{\mu l}$ & $\begin{array}{c}\text { Effectif } \\
(\mathbf{N}=\mathbf{7 3})\end{array}$ & $\begin{array}{c}\text { Fréquence } \\
(\boldsymbol{\%})\end{array}$ \\
\hline$<50$ & 13 & 17,8 \\
$50-199$ & 51 & 69,9 \\
$\geq 200$ & 9 & 12,3 \\
\hline
\end{tabular}

Environ 9/10 PV VIH étaient immunodéprimées à l'initiation au traitement ARV, 87,7\% dont 20,3\% étaient en état d'immunodépression sévère.

Tableau 3 : Répartition des PV VIH en fonction du taux de CD4 après quatre mois de traitement.

\begin{tabular}{lcc}
\hline $\begin{array}{l}\text { CD4 en } \\
\text { cellules/ } \boldsymbol{\mu l}\end{array}$ & Effectif $(\mathbf{N}=\mathbf{7 4})$ & $\begin{array}{c}\text { Fréquence } \\
(\boldsymbol{\%})\end{array}$ \\
\hline$<50$ & & 1,4 \\
$50-199$ & 1 & 18,9 \\
$\geq 200$ & 14 & 79,7
\end{tabular}

Le nombre d'immunodéprimés passe de $87,7 \%$ à $20,3 \%$ après quatre mois de traitement. Concomitamment (Tableau 5), après quatre mois de traitement, 50\% des PV VIH ayant à la fois le taux de Lymphocytes CD4 et la charge virale ont une charge virale indétectable.

Tableau 4 : Répartition des PV VIH en fonction du taux de lymphocytes T CD4 après douze mois de traitement.

\begin{tabular}{lcc}
\hline CD4 en cellules/ $\boldsymbol{\mu l}$ & Effectif $(\mathbf{N}=\mathbf{5 7})$ & $\begin{array}{c}\text { Fréquence } \\
\mathbf{( \% )}\end{array}$ \\
\hline$<50$ & 1 & 1,8 \\
$50-199$ & 7 & 12,2 \\
$\geq 200$ & 49 & 86,0 \\
\hline Le taux moyen de CD4 chez les PV VIH est passé de 137 cellules/ $\mu 1$ à l'initiation à 275 cellules/ $\mu 1$ à quatre \\
mois puis est passé à 371 cellules/ $\mu 1$ après douze mois de traitement ; soit une augmentation de $271 \%$.
\end{tabular}

Tableau 5 : Répartition des PV VIH en fonction de la charge virale après quatre mois de traitement.

\begin{tabular}{lcc}
\hline $\begin{array}{l}\text { Charge virale en } \\
\text { copies/ } \mathbf{m l}\end{array}$ & $\begin{array}{c}\text { Effectif } \\
(\mathbf{N = 1 8 )}\end{array}$ & $\begin{array}{c}\text { Fréquence } \\
(\boldsymbol{\%})\end{array}$ \\
\hline$<50$ & 9 & 50,0 \\
$50-500000$ & 9 & 50,0 \\
$>500000$ & 0 & 0,0 \\
\hline
\end{tabular}


Tableau 6 : Répartition des PVVIH en fonction de la charge virale après douze mois de traitement.

\begin{tabular}{|c|c|c|}
\hline $\begin{array}{l}\text { Charge virale } \\
\text { (copies/ ml) }\end{array}$ & $\begin{array}{l}\text { Effectif } \\
(\mathrm{N}=24)\end{array}$ & $\begin{array}{c}\text { Fréquence } \\
\qquad(\%)\end{array}$ \\
\hline$<50$ & 20 & 83,3 \\
\hline $50-500000$ & 4 & 16,7 \\
\hline$>500000$ & 0 & 0,0 \\
\hline
\end{tabular}

\section{DISCUSSION}

Les patients de la tranche 25-45 ans constituaient $70,7 \%$ de l'échantillon (Tableau 1) avec une médiane de 36,34 ans et $62,2 \%$ de femmes. Mouhari-Touré et al. (2011) dont l'objectif d'étude était de déterminer le profil immunologique et les anomalies biologiques des adultes à l'initiation du traitement antirétroviral ont trouvé des résultats similaires dans leur étude au Togo avec une médiane à 35 ans mais une prédominance féminine à $68,6 \%$. Une autre étude menée au Togo (Potchoo et al., 2010) dont le but était d'évaluer l'adhérence des PV VIH au traitement antirétroviral montrait une médiane d'âge similaire à la nôtre $(36,8 \%)$ et la même tranche d'âge la plus touchée par l'infection, mais avec un pourcentage plus élevé : $83,7 \%$ contre $70,7 \%$ dans notre étude. La prédominance du mode de transmission hétérosexuelle dans les régions tropicales, particulièrement en Afrique subsaharienne (Aubry, 2012), peut expliquer la prévalence de la maladie dans cette tranche d'âge. La sexratio est en général voisine de l'unité, ce qui permet de dire que la transmission de l'infection à VIH est hétérosexuelle et bidirectionnelle (Aubry, 2012); cette observation ne semble pas être vérifiée ni dans notre étude ni dans celle de Potchoo et al. (2010) dans lesquelles la sex-ratio en faveur des femmes était de 1,6 et de 0,3 , respectivement. $64,6 \%$ des sujets de notre série étaient observants. L'étude de Potchoo et al. (2010) et celle de Kebede et Wabe (2012) évaluaient l'adhérence au traitement et ses déterminants chez des PV VIH/SIDA et des PV VIH/SIDA coinfectées par le bacille tuberculeux, respectivement. Dans le premier groupe, $56 \%$ des patients étaient observants tandis que $95,8 \%$ de ceux du second groupe l'étaient en ce qui concerne uniquement la médication anti-VIH. Plusieurs auteurs ont étudié les causes de l'observance ou de la non observance en Afrique. Bien que l'adhérence au traitement et ses déterminants ne fussent pas l'objet de notre étude, les principaux déterminants (Potchoo et al., 2010 ; Sahali et al., 2011 ; Aubry, 2012 ; Kebede et al., 2012) de la non observance peuvent se résumer, d'après des publications récentes, par l'oubli, le coût du traitement, l'éloignement du centre de traitement ou les effets secondaires du traitement. Les prochaines études permettront de comprendre les causes de la non observance chez les malades du centre objet de notre étude. 95,1\% des PV VIH de notre échantillon ont été infectées par le VIH 1. Une étude togolaise incluant plus de cinq mille PV VIH à l'initiation du traitement ARV (Mouhari-Touré et al., 2011), comptait un pourcentage similaire au nôtre : $97,5 \%$ de personnes infectées par le VIH1. Le nombre moyen de lymphocytes CD4 à l'initiation du traitement des patients de l'étude de MouhariTouré et al. (2011) était de 134 cellules/ $\mu 1$ contre 137 cellules/ $\mu 1$ dans notre étude. Le gain moyen en lymphocytes CD4 des patients de notre étude (résultats : commentaires Tableaux 4 et 6 ) était de $271 \%$ entre l'initiation des ARV et les douze mois suivants. Les objectifs de nos deux études 
étaient différents. L'étude de Mouhari-Touré et al. (2011) déterminait le profil immunologique et les anomalies biologiques à l'initiation des ARV, la charge virale des patients n'a donc pas été déterminée. Leur cohorte comprenait 95,9\% d'immunodéprimés moyens et sévères contre $87,7 \%$ dans la nôtre. Bien que les objectifs respectifs des études citées dans notre discussion fussent différents, il ressort néanmoins que l'observance au traitement ARV était élevée en dépit de l'existence de déterminants à la non observance. Aubry (2012) dans son traité sur l'infection VIH/SIDA en zones tropicales estime que l'observance dans les pays de ces zones peut être aussi élevée qu'en pays industrialisé à condition de revoir la répercussion du coût du transport sur celui réel du traitement. Il ressort également de ces comparaisons que l'initiation aux traitements ARV est tardive en Ethiopie, au Togo comme au Bénin, d'où l'immunodépression sévère d'une proportion importante de patients à l'initiation du traitement. Le nombre de malades traités précocement peut augmenter grâce à l'amélioration des stratégies de dépistage de masse. Parallèlement à cette restauration des fonctions $\mathrm{du}$ système immunitaire obtenue dans notre étude, 50\% des sujets traités, possédant les deux valeurs (taux de CD4 et charge virale), passaient à une charge virale indétectable (Tableau 5). Ce succès virologique obtenu pourrait s'expliquer par l'adhésion au traitement de 64,6\% notée au sein des personnes traitées. Dans l'ensemble de la population étudiée, de bonnes réponses immunologiques et virologiques ont été obtenues. Ceci peut s'interpréter par l'efficacité des traitements antirétroviraux en cas de bonne observance thérapeutique. Quant à la relation entre les statuts immunologiques et virologiques des patients ; la distribution des PV VIH de notre série en fonction du taux des CD4 et la charge virale n'a pas été significative mais a permis d'établir la corrélation entre ces deux paramètres à partir d'un coefficient de corrélation compris entre -1 et 1 . Cette corrélation est d'autant plus forte que le coefficient s'éloigne davantage de zéro. Ainsi, ces coefficients de corrélation recherchés après quatre et douze mois de traitement antirétroviral sont respectivement de - 0,50 et 0,11 ; ce qui permet de dire que lorsque le taux de CD4 augmente la charge virale diminue. Ce coefficient $r=0,11$ après un an de traitement est peu significatif et pourrait s'améliorer si les patients adhéraient plus au traitement. Un coefficient de corrélation significatif qui cesse de l'être au cours du traitement peut être l'indication d'une diminution de l'efficacité du traitement suite à une mauvaise observance ou d'un début de résistance du VIH aux molécules utilisées. Son utilisation dans le suivi thérapeutique peut donc être important et permettre le réajustement des molécules utilisées pour le patient avant le constat d'un échec thérapeutique total. L'intérêt de pouvoir disposer d'un tel coefficient, à partir de ces deux examens, dans le suivi du traitement des PV VIH est appréciable.

\section{Conclusion}

La présente étude avait pour objectif de mesurer l'impact des traitements antirétroviraux d'importation sur la charge virale et sur le taux des lymphocytes CD4 des PV VIH en phase SIDA de l'hôpital de Tanguiéta au Bénin. Deux examens ont été nécessaires pour le suivi. Ce sont la numération des lymphocytes CD4 et la mesure de la charge virale. Au terme de cette étude, il ressort que lorsque le taux des lymphocytes CD4 augmente, la charge virale diminue. Au quatrième mois, le coefficient $r$ est égal à - 0,50 et permet de conclure que les ARV d'importation administrés à la population d'étude cible ont une efficacité. Il ressort également que ce coefficient de corrélation entre ces deux paramètres peut cesser d'être significatif au cours du traitement, et permettre avant l'échec thérapeutique total, un réajustement du traitement administré. Pour finir, le coefficient de corrélation $r$ entre le taux de lymphocytes 
CD4 et la charge virale des PV VIH sous traitement ARV peut permettre de mesurer l'efficacité du traitement antirétroviral administré, qu'il s'agisse des molécules d'importation ou des molécules proposées par les pharmacopées locales.

\section{REFERENCES}

Aubry P. 2012. Infection à VIH/SIDA et tropiques, actualités 2012. In Cours de Médecine Tropicale: Pathologie Infectieuse et Tropicale (en ligne). http://www. medecinetropicale.com/

Danel C, Moh R, Badje A, Ouattara E, Gabillard D, Ntakpe JB, Bohoussou F, Le Carrou J, Menan H, Anglaret X, Eholié S. 2012. Quand commencer le traitement antirétroviral en Afrique Sub-saharienne? Etat d'avancement de l'essai temprano ANRS 12136. http://www.vihgeneve2012 .com/programme/programmeComplet.asp ?DATE=26/03/2012.

Ford N. 2012. Commentary: The past, present and future of affordable antiretroviral therapy in Africa. Int. J. Epidemiol., 41(2): 460-461.

Ghosn J, Viard J-P. 2012. Le traitement personnalisé de l'infection par le VIH en 2012. Médecine Thérapeutique, 18(1): 310.

Kebede A, Tajure WN. 2012. Medication adherence and its determinants among patients on concomitant tuberculosis and antiretroviral therapy in South West Ethiopia. North American Journal of Medical Sciences, 4(2): 67-71.

Mouhari-Touré A, Patassi A, Nabroulaba KT, Djadou KE, Edou K, Nyametso D, Aho K, Saïbou A, Kombaté M, Kpanla K, Niman KW, Togbossi A, Agodomou E,
Wotogbe A, Tadona M, Singo A, Déku K, Pitche P. 2011. Profil biologique des patients adultes infectés par le VIH à l'initiation du traitement antirétroviral au Togo. Médecine et Maladies Infectieuses, 41(5): 229-234.

MSP/PNLS. 2004. Politiques, Normes et Procédures pour la Prise en Charge des IST au Benin. MSP/PNLS: Benin; 14-16.

Oette M, Schülter E, Rosen-Svi M, Peres Y, Zazzi M, Sönnerborg A, Struck D, Altmann A, Kaiser R, EuResist Network Study Group. 2012. Efficacy of antiretroviral therapy switch in HIV-infected patients: a ten years analysis of the EuResist Cohort. Intervirology, 55(2): 160-166.

Potchoo Y, Tchamdja K, Balogou A, Pitche VP, Guissou IP, Kassang EK. 2010. Knowledge and adherence to antiretroviral therapy among adult people living with HIV/AIDS treated in the health care centers of the association "Espoir Vie Togo" in Togo, West Africa. BMC Clinical Pharmacology, 10: 10.

Sahali S, Carcelain G, Goujard C, Delfraissy J-F, Ghosn J. 2011. Stratégies de restauration immunitaire chez les patients infectés par le virus de l'immunodéficience humaine. La Revue de Médecine Interne, 32(7): 425-431.

Stringer JSA, Mwango AJ, Giganti MJ, Mulenga L, Levy JW, Stringer EM, Mulenga P, Saag MS, Musonda P, williams FB, Reid SE, Chi BH. 2012. Effectiveness of generic and proprietary first-line anti-retroviral regimens in a primary health care setting in Lusaka, Zambia: a cohort study. Int. J. Epidemiol., 41(2): 448-459. 